\title{
Evaluation of glycemic traits in susceptibility to COVID-19 risk: a Mendelian randomization study
}

\author{
Shiu Lun Au Yeung ${ }^{1 *}$, Jie V Zhao ${ }^{1}$ and C Mary Schooling ${ }^{1,2}$
}

\begin{abstract}
Background: Observational studies suggest poorer glycemic traits and type 2 diabetes associated with coronavirus disease 2019 (COVID-19) risk although these findings could be confounded by socioeconomic position. We conducted a two-sample Mendelian randomization to clarify their role in COVID-19 risk and specific COVID-19 phenotypes (hospitalized and severe cases).

Method: We identified genetic instruments for fasting glucose $(n=133,010), 2 \mathrm{~h}$ glucose $(n=42,854)$, glycated hemoglobin $(n=123,665)$, and type 2 diabetes $(74,124$ cases and 824,006 controls) from genome wide association studies and applied them to COVID-19 Host Genetics Initiative summary statistics (17,965 COVID-19 cases and 1,370, 547 population controls). We used inverse variance weighting to obtain the causal estimates of glycemic traits and genetic predisposition to type 2 diabetes in COVID-19 risk. Sensitivity analyses included MR-Egger and weighted median method.
\end{abstract}

Results: We found genetic predisposition to type 2 diabetes was not associated with any COVID-19 phenotype (OR: 1.00 per unit increase in log odds of having diabetes, $95 \% \mathrm{Cl} 0.97$ to 1.04 for overall COVID-19; OR: 1.02, 95\%Cl 0.95 to 1.09 for hospitalized COVID-19; and OR: 1.00, 95\%Cl 0.93 to 1.08 for severe COVID-19). There were no strong evidence for an association of glycemic traits in COVID-19 phenotypes, apart from a potential inverse association for fasting glucose albeit with wide confidence interval.

Conclusion: We provide some genetic evidence that poorer glycemic traits and predisposition to type 2 diabetes unlikely increase the risk of COVID-19. Although our study did not indicate glycemic traits increase severity of COVID-19, additional studies are needed to verify our findings.

Keywords: COVID-19, Glucose, Glycated hemoglobin, Mendelian randomization, Type 2 diabetes

\section{Background}

Coronavirus disease 19 (COVID-19) has become a major global health threat. While randomized controlled trials have been quickly conducted to identify possible treatments for COVID-19 [1], other observational studies focus on factors related to increased susceptibility to

\footnotetext{
* Correspondence: ayslryan@hku.hk

${ }^{1}$ School of Public Health, LKS Faculty of Medicine, The University of Hong Kong, 1/F, Patrick Manson Building, 7 Sassoon Road, Hong Kong, SAR, China Full list of author information is available at the end of the article
}

COVID-19 risk or its severity. Hyperglycemia and type 2 diabetes diagnosis have been associated with increased risk of COVID-19 or with complications in previous observational studies [2-5]. However, it is unclear whether these associations indicate causal targets of intervention due to the possibility of confounding and bias. Furthermore, these observational studies primarily used patient data, and hence, it is unclear whether the observed associations apply to non-hospitalized cases, i.e., less severe

C C The Author(s). 2021 Open Access This article is licensed under a Creative Commons Attribution 4.0 International License, which permits use, sharing, adaptation, distribution and reproduction in any medium or format, as long as you give appropriate credit to the original author(s) and the source, provide a link to the Creative Commons licence, and indicate if changes were made. The images or other third party material in this article are included in the article's Creative Commons licence, unless indicated otherwise in a credit line to the material. If material is not included in the article's Creative Commons licence and your intended use is not permitted by statutory regulation or exceeds the permitted use, you will need to obtain permission directly from the copyright holder. To view a copy of this licence, visit http://creativecommons.org/licenses/by/4.0/ The Creative Commons Public Domain Dedication waiver (http://creativecommons.org/publicdomain/zero/1.0/) applies to the data made available in this article, unless otherwise stated in a credit line to the data. 
and likely asymptomatic cases which may account for the majority of the COVID-19 cases [6].

The use of Mendelian randomization studies helps circumvent the limitations of previous observational studies due to its resistance to confounding by the use of genetic variants randomly allocated at conception to proxy exposures [7]. A recent Mendelian randomization study suggested a role of diabetes in increased ACE2 lung expression, the receptor for SARS-CoV-2 [8], and hence an increased risk of COVID-19. However, an association with a "surrogate endpoint", i.e., ACE2 expression, does not always indicate a causal effect on the outcome, here COVID-19. To address the causal role of glycemic traits and type 2 diabetes in COVID-19, we conducted a Mendelian randomization study using summary statistics from relevant genome wide association studies (GWAS) of glycemic traits, type 2 diabetes, and COVID-19 [913]. We also included subtypes of COVID-19 phenotypes, including hospitalized cases and severe cases, as previous studies also used these more severe COVID-19 phenotypes as outcomes.

\section{Methods}

This is a 2 sample Mendelian randomization study which used summary statistics from relevant GWAS [7]. There are three assumptions. First, the genetic instruments predict the exposure. Second, the genetic instruments are independent of confounders of the exposureoutcome relation; this assumption has been demonstrated empirically in previous studies [14]. Third, the genetic instruments' effect on the outcome, if any, is only via its relation with the exposure, here glycemic traits and type 2 diabetes.

\section{Exposure GWAS-glycemic traits and type 2 diabetes}

We extracted genetic instruments, i.e., single nucleotide polymorphism (SNP), for glycemic traits from MAGIC GWAS summary statistics, one of the largest genetic consortia on glycemic traits where these GWAS were conducted among people without diabetes. We restricted the sample to people of European descent, and selected instruments strongly ( $p$ value $<5 \times 10^{-8}$ ) and independently (not in linkage disequilibrium (LD, $r^{2}<0.001$ based on European population reference panel) associated with the phenotypes. Specifically, instruments for fasting glucose were obtained from a GWAS of up to 133,010 participants [10]. Instruments for $2 \mathrm{~h}$ glucose were obtained from a GWAS of up to 42,854 participants [10]. Instruments for glycated hemoglobin (HbA1c) were obtained from a GWAS of 123,665 participants of European ancestry [13], out of the original GWAS comprised of 159, 940 participants of mixed ancestries, as previously [15]. Mean age in these GWAS was around 50 years, with similar proportion of men and women. These GWAS adjusted for age and sex, study-specific covariates, and used genomic control.

Instruments for type 2 diabetes were obtained from the DIAMANTE consortium ( $p$ value $<5 \times 10^{-8}$ and not in LD $\left.\left(\mathrm{r}^{2}<0.001\right)\right)$, one of the largest GWAS consortia on type 2 diabetes [12], which comprised 898,130 participants $(74,124$ cases and 824,006 controls) of European descent [12]. Type 2 diabetes was defined in multiple ways, such as fasting glucose $(\geq 7.0 \mathrm{mmol} / \mathrm{L})$, previous diagnosis of type 2 diabetes, case ascertainment from electronic health records, and use of anti-diabetic medication. The mean age of cases was around 55 years. The GWAS had similar proportions of men and women. The GWAS adjusted for study-specific covariates and controlled for population stratification with genomic control to reduce confounding by ethnicity.

Additional file 1: Table S1 shows the list of genetic instruments used in this study. We calculated the variance of the exposures explained by each instrument using established equations for continuous and binary exposures $[16,17]$, as shown in Additional file 1: Table S1.

\section{Outcome GWAS-COVID-19 phenotypes}

We extracted summary statistics of all three COVID-19 phenotypes from the most recent data freeze at the time of analyses (Round 4, October 2020) in COVID-19 Host Genetics Initiative (www.covid19hg.org), accessed on November 3, 2020 [11], using rs number or position (Genome Reference Consortium Human Build 37). The COVID-19 Host Genetics Initiative is an initiative comprised of several epidemiologic studies of various designs, such as UK Biobank, deCODE, and FinnGen but we excluded 23andMe study given data from this study were not included in the summary statistics. These phenotypes included any COVID-19 cases, hospitalized COVID-19 cases, and severe COVID cases. In brief, any COVID-19 was defined as having laboratory-confirmed SARS-CoV-2 infection, confirmed COVID-19 from electronic health records/doctor diagnosis, or self-reported COVID-19 positive (17,965 cases and 1,370,547 population controls). Hospitalized COVID was defined as having hospitalized laboratory-confirmed SARS-CoV-2 infection or hospitalization due to corona-related symptom (7885 cases and 961,804 population controls without COVID-19). Very severe respiratory confirmed COVID-19 was defined as having hospitalized laboratory-confirmed SARS-CoV-2 infection, with respiratory support or death (4336 cases and 623,902 population controls without COVID-19). The GWAS was adjusted for sex, age, age ${ }^{2}$, age ${ }^{*}$ sex, principal components and study-specific covariates. Additional file 1: Table S2 shows the instruments' association with COVID-19 phenotypes. Details of COVID-19 Host Genetics Initiative, such as case ascertainment and contributing studies can be found in Additional file 1: Tables S3-S6. 


\section{Exposures}

The exposures were HbAlc (\%), fasting glucose (mmol/ $\mathrm{L}), 2 \mathrm{~h}$ glucose $(\mathrm{mmol} / \mathrm{L})$, and predisposition to type 2 diabetes (per unit increase in log odds of having diabetes).

\section{Outcomes}

The primary outcome was any COVID-19 cases. The secondary outcomes were hospitalized COVID-19 and severe COVID-19 cases.

\section{Pleiotropic effects}

Given a previous Mendelian randomization study suggested a possible role of body mass index (BMI) and smoking on risk of severe COVID-19, we explored the association of genetic instruments of glycemic traits and predisposition to type 2 diabetes in respective genome wide association studies (GIANT consortium for BMI and GSCAN for cigarettes smoked per day) [18, 19], where we considered evidence of pleiotropy if the SNP association reached genome wide significance ( $p$ value $<$ $\left.5 \times 10^{-8}\right)$.

\section{Statistical analyses}

We approximated the F statistics of each instrument to assess potential weak instrument bias, where higher $F$ statistics indicated lower risk of weak instrument bias [20]. We assessed the association of genetically predicted glycemic traits and predisposition to type 2 diabetes with COVID-19 phenotypes using inverse variance weighting with multiplicative random effects. We also reported heterogeneity of the Wald ratios, i.e., the genetic association with the outcome divided by the genetic association with the exposure, from the Cochrane Q statistics and the MR-Egger intercept $p$ value as indicators of potential pleiotropy of the included instruments. We conducted sensitivity analyses using the weighted median and MR-Egger [21, 22].

\section{Power calculation}

We calculated the variance of the exposures explained by each instrument using an established approximation for continuous and binary exposures [16, 17], as shown in Additional file 1: Table S1. We estimated power using the approximation that the sample size for an MR study is the sample size for exposure on outcome divided by the variance of the exposures [23, 24], as shown in Additional file 1: Table S7.

All analyses were performed using $\mathrm{R}$ Version 3.6.1 ( $\mathrm{R}$ Core Team (2019). R: A language and environment for statistical computing. R Foundation for Statistical Computing, Vienna, Austria. https://www.R-project.org/) and the R package (“TwoSampleMR”) [9].

\section{Ethics approval}

This study only used publicly available data and hence no ethics approval was required.

\section{Results}

Up to 34 genetic instruments for HbA1c, 33 instruments for fasting glucose, 7 instruments for $2 \mathrm{~h}$ glucose, and 156 instruments for predisposition to type 2 diabetes were used in this study. All instruments had an F statistic >10, implying weak instrument bias is less likely (Additional file 1: Table S1). Forty-two (42) SNPs were associated with BMI and none of the SNPs were associated with cigarettes smoked per day. Hence, these SNPs were excluded in the sensitivity analyses.

Figure 1 shows the association of genetically predicted glycemic traits and genetic predisposition to type 2 diabetes on risk of COVID-19. There was no strong evidence for an effect of glycemic traits on COVID-19 risk, apart from fasting glucose, where higher fasting glucose appeared to be associated with lower risk of being a COVID-19 case although the estimates had wide confidence intervals. Higher predisposition to type 2 diabetes was not associated with COVID-19 risk, with estimates close to null. The Cochrane $\mathrm{Q}$ statistics test and MREgger intercept test where there was no strong evidence for heterogeneity, apart from HbA1c where there were signs of horizontal pleiotropy based on the MR-Egger intercept ( $p$ value: 0.004). However, the corresponding estimates from MR-Egger and weighted median were not always consistent with the main analyses, but with wide confidence intervals. Figures 2 and 3 show the association of genetically predicted glycemic traits and genetic predisposition to type 2 diabetes on risk of hospitalized COVID-19 and severe COVID-19, which indicated no strong evidence for an effect of glycemic traits and predisposition to type 2 diabetes on these more severe COVID-19 phenotypes. There were signs of pleiotropy in some of the analyses, such as for predisposition to type 2 diabetes (Cochrane Q test $p$ value: 0.004 ) related to hospitalized COVID-19 and for HbA1c related to severe COVID-19 (Cochrane $Q$ test $p$ value: 0.04 ).

Exclusion of SNPs related to BMI did not change the conclusion regardless of the COVID-19 phenotypes (Additional file 1: Figures S1-S3).

\section{Discussion}

This Mendelian randomization study provides potentially more credible evidence concerning the role of glycemic traits and predisposition to type 2 diabetes in COVID-19 risk and provides no strong evidence for a causal role of hyperglycemia in increasing risk of COVID-19 or its severity. In particular, the estimates for genetic predisposition to type 2 diabetes were close to null. This is consistent with a previous Mendelian 


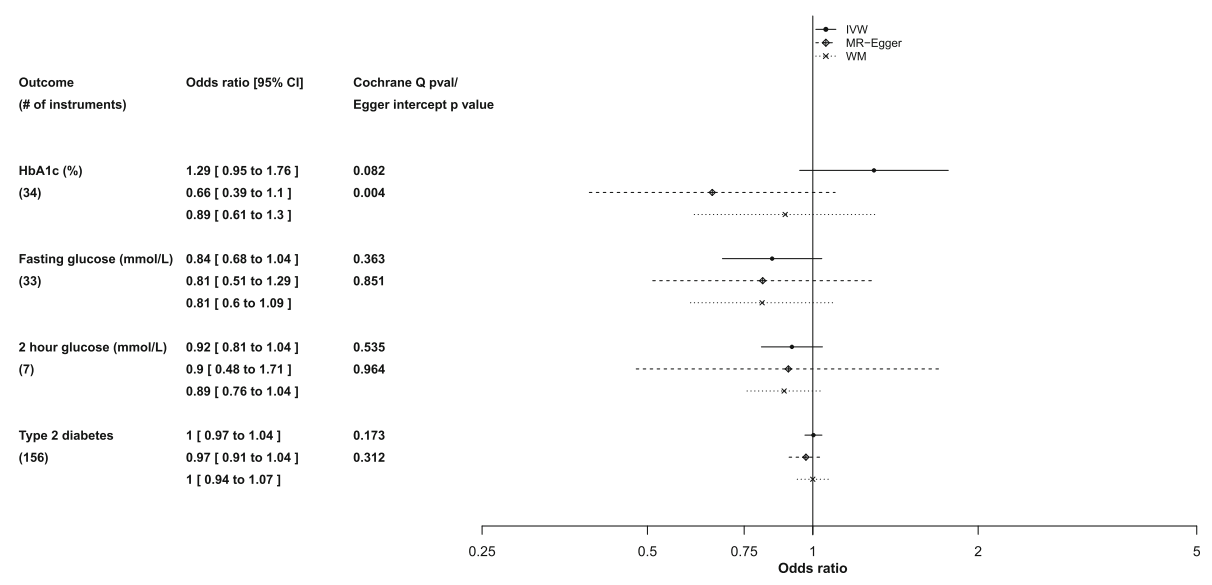

Fig. 1 Association of genetically predicted glycemic traits and genetic predisposition to type 2 diabetes on risk of COVD-19 using Mendelian randomization

randomization study using data from the previous data freeze (Release 3, June 2020) with smaller sample size for hospitalized and severe COVID-19 cases [25]. Our study adds by showing no strong evidence for an association of glycemic traits in these COVID-19 phenotypes using larger case numbers. Furthermore, our study indicated that glycemic traits and predisposition to type 2 diabetes unlikely have a strong role in increasing susceptibility to overall COVID-19, which may be more relevant to the general population given the majority of COVID-19 cases were not severe [26].

Exploring underlying causes of COVID-19 is important for efficient allocation of public health resources to reduce related infections in the population. The recent OpenSAFELY study, using electronic health records of more than 17 million people in the UK, showed that older age, being a male, being obese, lower socioeconomic position, and diabetes were associated with higher risk of COVID-19 related death [4]. Although some of these factors may be causal, such as higher body mass index, some may be only risk factors, such as diabetes [25], perhaps because of its association with lower socio-economic position. Furthermore, some findings could be a reflection of bias, such as the paradoxically lower risk of COVID-19 deaths among current smokers, which was not supported by Mendelian randomization which showed a detrimental effect of smoking on COVID-19 [25]. Nevertheless, observational studies provide insights regarding potential causes of COVID-19 but the findings should be further validated using Mendelian randomization when more severe cases have accumulated in the COVID-19 Host Genetics Initiative or using genetic risk scores in the UK Biobank to maximize statistical power [27]. It is also possible that glycemic traits/type 2 diabetes may affect risk of COVID-19 in specific subgroups, perhaps related to underlying genetic susceptibility to COVID-19. However, these dimensions of vulnerability remain to be identified.

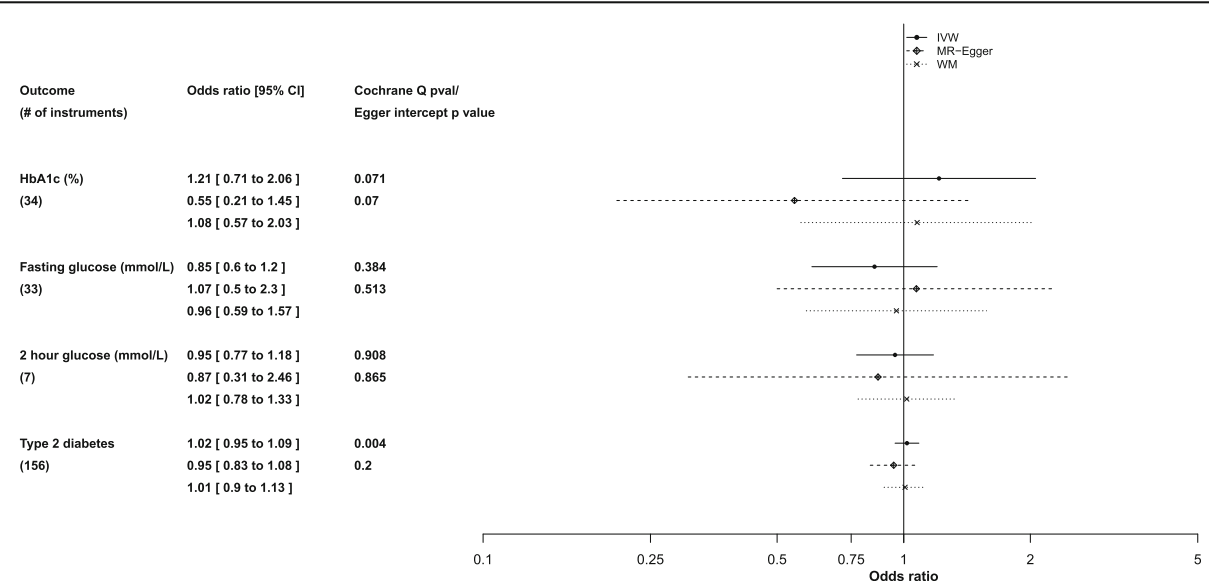

Fig. 2 Association of genetically predicted glycemic traits and genetic predisposition to type 2 diabetes on risk of hospitalized COVID-19 using Mendelian randomization 


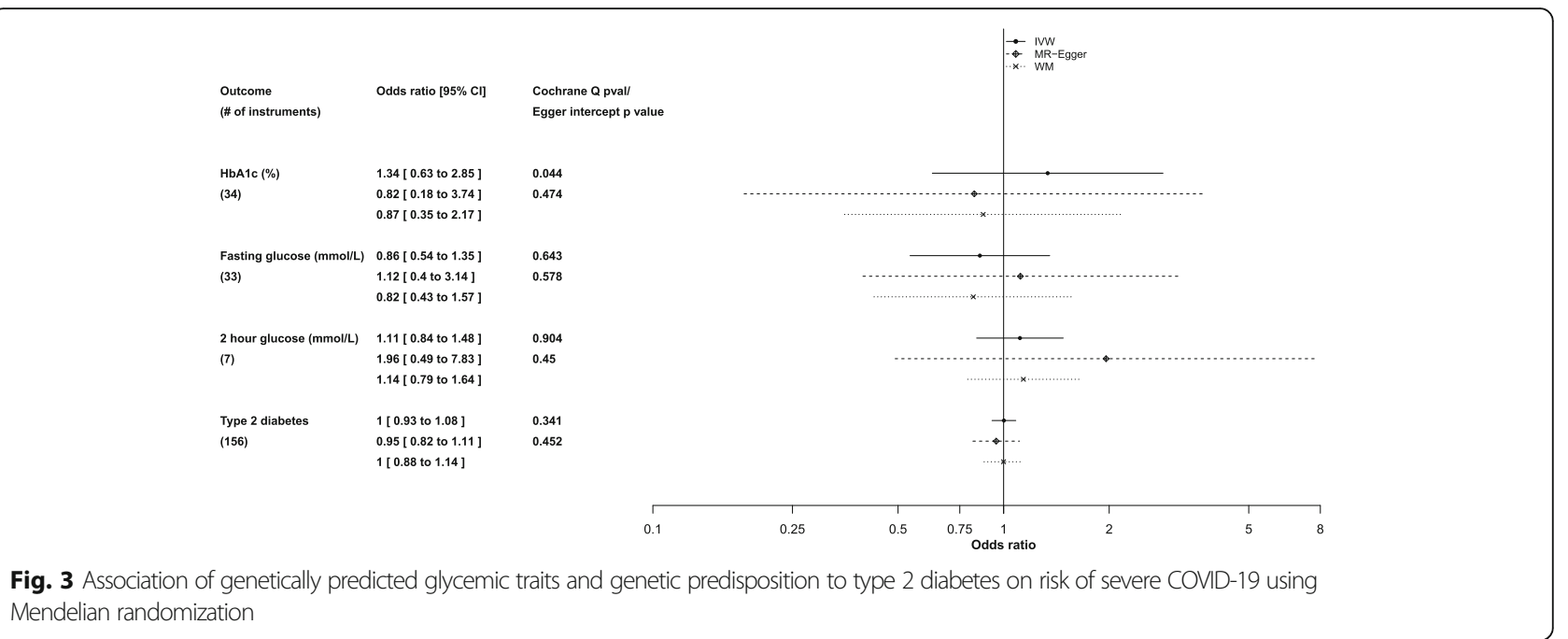

One of the motivating reasons for this study is that a previous Mendelian randomization study suggested a possible role of type 2 diabetes in increased risk of COVID-19 [8]. However, that study only used ACE2 expression in the lung as a surrogate outcome, and may not necessarily translate to an effect on the actual outcome, i.e., SARS-CoV-2 infection. This difference has been demonstrated by the debates related to ACE inhibitors where concerns were raised over its use given the possibility of increasing ACE2 expression [28] although subsequent evidence suggests that ACE inhibitors do not appear to increase the risk of COVID-19 [29].

Previous observational studies suggested a possible link of diabetes in infection risk due to possible impairment on the immune system [30,31], and hence may be relevant to COVID-19 susceptibility and its severity [25]. However, the discrepancies between these findings and our study may be indicative of confounding by obesity, or perhaps by medications such as metformin use which were potentially related to lower mortality among those who were admitted to hospital due to COVID-19 [32]. This would warrant further investigations such as using relevant genetic instruments for these medications to assess their impact on COVID-19 severity [33].

Although we used Mendelian randomization which is more resistant to confounding, there are some limitations. Mendelian randomization relies on assumptions for valid inference [7]. We used genetic instruments extracted from published GWAS to reduce risk of weak instrument bias. There was some evidence for pleiotropy in our analyses, indicating the inverse variance weighting analyses may not be valid although the conclusion from MR-Egger remains unchanged. These SNPs do not appear to affect infectious disease risk based on PhenoScanner, a curated database on genetic effects [34], although some SNPs were related to immune markers such as eosinophil and lymphocytes [35] while other SNPs were related to BMI. Nevertheless, pleiotropic effects may have partly explained why estimates from sensitivity analyses were not always directionally consistent for these analyses. Our findings should be verified in future studies when larger number of cases accumulated in the COVID-19 genetic consortium. Mendelian randomization studies are also vulnerable to selection bias and this may partly explain the potential "inverse" association of glucose with COVID phenotypes due to potential competing risk of survival [36]. In this study, we assessed genetic predisposition of type 2 diabetes in COVID-19 phenotypes, and hence, we were not able to assess the impact of type 2 diabetes diagnosis on COVID-19 susceptibility as many of the participants in the COVID-19 GWAS may not have developed type 2 diabetes [37]. As such, the null findings did not directly rule out the possibility of a role of type 2 diabetes in COVID-19 susceptibility. However, the inconsistent findings across glycemic traits and genetic predisposition to type 2 diabetes may suggest hyperglycemia per se did not appear to have a role in susceptibility to COVID-19 risk and its severity. There are also challenges regarding the identification of cases in the course of this COVID19 pandemic. For example, some of the controls could potentially be "cases" which were not identified given the majority of the cases are asymptomatic or because they were not being tested because of particular social distancing policies targeting high risk groups. This misclassification would also inevitably bias our estimates towards null [6] although this is a challenge also applicable to other COVID-19 studies such as estimation of the case fatality risk [38]. Furthermore, case ascertainment methods were not the same in different cohorts (Additional file 1: Tables S3-S6) but we were unable to explore how these differences may influence our findings 
because we only had access to overall summary statistics. Lastly, our case number for secondary outcomes was not high and hence we could not rule out the possibility of smaller effects of glycemic traits/ type 2 diabetes on COVID-19 risk. For example, regarding HbA1c, the detectable effect size for per change in standard deviation of HbA1c $(\sim 0.6 \%$ based on our previous study in UK Biobank [33]), would be an odds ratio of 1.15 for any COVID-19 cases, 1.25 for hospitalized COVID-19 cases, and 1.35 for severe COVID-19 cases).

\section{Conclusions}

Our Mendelian randomization study suggested glycemic traits and type 2 diabetes do not appear to increase the risk of COVID-19. As such, our study may imply the observed associations of diabetes with COVID-19 may be at least partly due to people with diabetes being vulnerable to COVID-19 for structural reasons, such as precarious employment or crowded housing [39]. Our study also showed that there is no clear evidence of glycemic traits and type 2 diabetes in increasing the risk of more severe COVID-19. However, these findings should be replicated when larger genetic studies become available.

\section{Supplementary Information}

The online version contains supplementary material available at https://doi. org/10.1186/s12916-021-01944-3.

Additional file 1: Table S1. Genetic instruments used in this Mendelian randomization study. Table S2. Genetic instruments' associations with COVID-19 phenotypes. Table S3. Studies related to COVID-19 analysis, as per extracted from COVID-19 Host Genetics Initiative (https://www.covid1 9hg.org/). Table S4. Studies related to hospitalized COVID-19 analysis, as per extracted from COVID-19 Host Genetics Initiative (https://www.covid1 9hg.org/). Table S5. Studies related to severe COVID-19 analysis, as per extracted from COVID-19 Host Genetics Initiative (https://www.covid19hg. org/). Table S6. Definition of the COVID-19 phenotypes, as per extracted from COVID-19 Host Genetics Initiative (https://www.covid19hg.org/). Table S7. Minimum detectable odds ratio per standard deviation of the exposure in this Mendelian randomization, at 80\% power and 5\% statistical significance. Figure S1. Association of genetically predicted glycemic traits and genetic predisposition to type 2 diabetes on risk of COVID-19 using Mendelian randomization, excluding instruments related to body mass index. Figure S2. Association of genetically predicted glycemic traits and genetic predisposition to type 2 diabetes on risk of hospitalized COVID-19 using Mendelian randomization excluding instruments related to body mass index. Figure $\mathbf{S 3}$. Association of genetically predicted glycemic traits and genetic predisposition to type 2 diabetes on risk of severe COVID-19 using Mendelian randomization excluding instruments related to body mass index.

\section{Acknowledgements}

We thank the COVID-19 Host Genetics Initiative (https://www.covid19hg.org/ ) for making the summary statistics of COVID-19 phenotypes publicly available. We thank the MAGIC consortium (https://www.magicinvestigators.org/) for making the summary statistics of glycemic traits publicly available. We thank the GIANT consortium ("ieu-b-40") for making the summary statistics of body mass index, which were accessed via MR-Base. We thank GSCAN for making the summary statistics of cigarettes smoked per day available, which were downloaded from https://conservancy.umn.edu/handle/11299/201564. We thank Ms. Queenie Li for compiling the tables related to the COVID-19 Host Genetics Initiative.

\section{Authors' contributions}

SLAY designed the study, wrote the analysis plan, and interpreted the results. SLAY undertook analyses with feedback from JVZ and CMS. SLAY wrote the first draft of the manuscript with critical feedback and revisions from JVZ and CMS. All authors gave final approval of the version to be published.

\section{Funding}

This study is partly supported by the Research Output Prize 2020, LKS Faculty of Medicine, The University of Hong Kong.

\section{Availability of data and materials}

The data used in this study were publicly available and can be accessed via the links described in the Acknowledgement and the references in the manuscript.

Ethics approval and consent to participate

Not applicable.

Consent for publication

Not applicable.

Competing interests

None declared.

\section{Author details}

${ }^{1}$ School of Public Health, LKS Faculty of Medicine, The University of Hong Kong, 1/F, Patrick Manson Building, 7 Sassoon Road, Hong Kong, SAR, China. ${ }^{2}$ School of Public Health and Health Policy, City University of New York, New York, USA.

Received: 10 November 2020 Accepted: 16 February 2021

Published online: 24 March 2021

\section{References}

1. Recovery Collaborative Group, Horby P, Lim WS, Emberson JR, Mafham M, Bell JL, Linsell L, Staplin N, Brightling C, Ustianowski A, et al. Dexamethasone in hospitalized patients with Covid-19 - preliminary report. N Engl J Med. 2020. https://pubmed.ncbi.nlm.nih.gov/32678530/.

2. Wang S, Ma P, Zhang S, Song S, Wang Z, Ma Y, Xu J, Wu F, Duan L, Yin Z, et al. Fasting blood glucose at admission is an independent predictor for 28-day mortality in patients with COVID-19 without previous diagnosis of diabetes: a multi-centre retrospective study. Diabetologia. 2020;63:2102-11. https://pubmed.ncbi.nlm.nih.gov/32647915/.

3. Wu C, Chen X, Cai Y, Xia J, Zhou X, Xu S, Huang H, Zhang L, Zhou X, Du C, et al. Risk factors associated with acute respiratory distress syndrome and death in patients with coronavirus disease 2019 pneumonia in Wuhan, China. JAMA Intern Med. 2020;180:934-43. https://pubmed.ncbi.nlm.nih. gov/32167524/.

4. Williamson EJ, Walker AJ, Bhaskaran K, Bacon S, Bates C, Morton CE, Curtis HJ, Mehrkar A, Evans D, Inglesby P, et al. OpenSAFELY: factors associated with COVID-19 death in 17 million patients. Nature. 2020;584:430-6. https:// pubmed.ncbi.nlm.nih.gov/32640463/.

5. Richardson S, Hirsch JS, Narasimhan M, Crawford JM, McGinn T, Davidson KW, the Northwell C-RC, Barnaby DP, Becker LB, Chelico JD, et al. Presenting characteristics, comorbidities, and outcomes among 5700 patients hospitalized with COVID-19 in the New York City area. JAMA. 2020;323: 2052-9. https://pubmed.ncbi.nlm.nih.gov/32320003/.

6. COVID-19: What proportion are asymptomatic? [https://www.cebm.net/ covid-19/covid-19-what-proportion-are-asymptomatic/].

7. Davies NM, Holmes MV, Davey Smith G. Reading Mendelian randomisation studies: a guide, glossary, and checklist for clinicians. BMJ. 2018;362:k601.

8. Rao S, Lau A, So HC. Exploring diseases/traits and blood proteins causally related to expression of ACE2, the putative receptor of SARS-CoV-2: a Mendelian randomization analysis highlights tentative relevance of diabetes-related traits. Diabetes Care. 2020;43(7):1416-26.

9. Hemani G, Zheng J, Elsworth B, Wade KH, Haberland V, Baird D, Laurin C, Burgess S, Bowden J, Langdon R, et al. The MR-base platform supports systematic causal inference across the human phenome. Elife. 2018;7: e34408. https://pubmed.ncbi.nlm.nih.gov/29846171/.

10. Scott RA, Lagou V, Welch RP, Wheeler E, Montasser ME, Luan J, Magi R, Strawbridge RJ, Rehnberg E, Gustafsson S, et al. Large-scale association 
analyses identify new loci influencing glycemic traits and provide insight into the underlying biological pathways. Nat Genet. 2012;44(9):991-1005.

11. COVID-19 Host Genetics Initiative. The COVID-19 Host Genetics Initiative, a global initiative to elucidate the role of host genetic factors in susceptibility and severity of the SARS-CoV-2 virus pandemic. Eur J Hum Genet. 2020; 28(6):715-8.

12. Mahajan A, Taliun D, Thurner M, Robertson NR, Torres JM, Rayner NW, Payne AJ, Steinthorsdottir V, Scott RA, Grarup N, et al. Fine-mapping type 2 diabetes loci to single-variant resolution using high-density imputation and islet-specific epigenome maps. Nat Genet. 2018;50(11):1505-13.

13. Wheeler E, Leong A, Liu CT, Hivert MF, Strawbridge RJ, Podmore C, Li M, Yao J, Sim X, Hong J, et al. Impact of common genetic determinants of hemoglobin A1c on type 2 diabetes risk and diagnosis in ancestrally diverse populations: a transethnic genome-wide meta-analysis. PLoS Med. 2017; 14(9):e1002383.

14. Davey-Smith G, Lawlor DA, Harbord R, Timpson N, Day I, Ebrahim S. Clustered environments and randomized genes: a fundamental distinction between conventional and genetic epidemiology. PLoS Med. 2007;4(12):e352.

15. Au Yeung SL, Luo S, Schooling CM. The impact of Glycated hemoglobin (HbA1c) on cardiovascular disease risk: a Mendelian randomization study using UK biobank. Diabetes Care. 2018;41(9):1991-7.

16. Yarmolinsky J, Bonilla C, Haycock PC, Langdon RJQ, Lotta LA, Langenberg C, Relton CL, Lewis SJ, Evans DM, Consortium P, et al. Circulating selenium and prostate Cancer risk: a Mendelian randomization analysis. J Natl Cancer Inst. 2018;110(9):1035-8.

17. Lee SH, Wray NR. Novel genetic analysis for case-control genome-wide association studies: quantification of power and genomic prediction accuracy. PLoS One. 2013;8(8):e71494.

18. Liu M, Jiang Y, Wedow R, Li Y, Brazel DM, Chen F, Datta G, Davila-Velderrain J, McGuire D, Tian C, et al. Association studies of up to 1.2 million individuals yield new insights into the genetic etiology of tobacco and alcohol use. Nat Genet. 2019:51(2):237-44.

19. Yengo L, Sidorenko J, Kemper KE, Zheng Z, Wood AR, Weedon MN, Frayling TM, Hirschhorn J, Yang J, Visscher PM, et al. Meta-analysis of genome-wide association studies for height and body mass index in approximately 700000 individuals of European ancestry. Hum Mol Genet. 2018:27(20):3641-9.

20. Bowden J, Del Greco MF, Minelli C, Davey Smith G, Sheehan NA, Thompson JR. Assessing the suitability of summary data for two-sample Mendelian randomization analyses using MR-Egger regression: the role of the 12 statistic. Int J Epidemiol. 2016;45:1961-74. https://pubmed.ncbi.nlm.nih. gov/27616674/.

21. Bowden J, Davey Smith G, Haycock PC, Burgess S. Consistent estimation in Mendelian randomization with some invalid instruments using a weighted median estimator. Genet Epidemiol. 2016;40(4):304-14.

22. Bowden J, Davey Smith G, Burgess S. Mendelian randomization with invalid instruments: effect estimation and bias detection through Egger regression. Int J Epidemiol. 2015;44(2):512-25.

23. Burgess S. Sample size and power calculations in Mendelian randomization with a single instrumental variable and a binary outcome. Int J Epidemiol. 2014;43(3):922-9.

24. Freeman G, Cowling BJ, Schooling CM. Power and sample size calculations for Mendelian randomization studies using one genetic instrument. Int J Epidemiol. 2013:42(4):1157-63.

25. Ponsford MJ, Gkatzionis A, Walker VM, Grant AJ, Wootton RE, Moore LSP, Fatumo S, Mason AM, Zuber V, Willer C, et al. Cardiometabolic traits, sepsis, and severe COVID-19: a Mendelian randomization investigation. Circulation. 2020;142(18):1791-3.

26. Petersen I, Phillips A. Three quarters of people with SARS-CoV-2 infection are asymptomatic: analysis of English household survey data. Clin Epidemiol. 2020;12:1039-43.

27. Sudlow C, Gallacher J, Allen N, Beral V, Burton P, Danesh J, Downey P, Elliott $P$, Green J, Landray $M$, et al. UK biobank: an open access resource for identifying the causes of a wide range of complex diseases of middle and old age. PLoS Med. 2015;12(3):e1001779.

28. Fang L, Karakiulakis G, Roth M. Are patients with hypertension and diabetes mellitus at increased risk for COVID-19 infection? Lancet Respir Med. 2020; 8(4):e21.

29. Fosbol EL, Butt JH, Ostergaard L, Andersson C, Selmer C, Kragholm K, Schou M, Phelps M, Gislason GH, Gerds TA, et al. Association of angiotensinconverting enzyme inhibitor or angiotensin receptor blocker use with
COVID-19 diagnosis and mortality. JAMA. 2020;324:168-77. https://pubmed. ncbi.n/m.nih.gov/32558877/.

30. Critchley JA, Carey IM, Harris T, DeWilde S, Hosking FJ, Cook DG. Glycemic control and risk of infections among people with type 1 or type 2 diabetes in a large primary care cohort study. Diabetes Care. 2018;41(10):2127-35.

31. Mor A, Dekkers OM, Nielsen JS, Beck-Nielsen H, Sorensen HT, Thomsen RW. Impact of glycemic control on risk of infections in patients with type 2 diabetes: a population-based cohort study. Am J Epidemiol. 2017;186(2): 227-36.

32. Bramante $C T$, Ingraham NE, Murray TA, Marmor S, Hovertsen S, Gronski J, McNeil C, Feng R, Guzman G, Abdelwahab N, et al. Metformin and risk of mortality in patients hospitalised with COVID-19: a retrospective cohort analysis. Lancet Healthy Longev. 2021;2(1):e34-41.

33. Luo S, Schooling CM, Wong ICK, Au Yeung SL. Evaluating the impact of AMPK activation, a target of metformin, on risk of cardiovascular diseases and cancer in the UK Biobank: a Mendelian randomisation study. Diabetologia. 2020;63(11):2349-58.

34. Staley JR, Blackshaw J, Kamat MA, Ellis S, Surendran P, Sun BB, Paul DS, Freitag D, Burgess S, Danesh J, et al. PhenoScanner: a database of human genotype-phenotype associations. Bioinformatics. 2016;32(20):3207-9.

35. Astle WJ, Elding $H$, Jiang T, Allen D, Ruklisa D, Mann AL, Mead D, Bouman H, Riveros-Mckay F, Kostadima MA, et al. The allelic landscape of human blood cell trait variation and links to common complex disease. Cell. 2016;167(5): $1415-29$ e1419.

36. Schooling C, Lopez P, Yang Z, Zhao JV, Au Yeung S, Huang J. Use of multivariable Mendelian randomization to address biases due to competing risk before recruitment. Front Genet. 2021;11:610852. https://pubmed.ncbi. nlm.nih.gov/33519914/.

37. Burgess S, Labrecque JA. Mendelian randomization with a binary exposure variable: interpretation and presentation of causal estimates. Eur J Epidemiol. 2018;33(10):947-52.

38. Wu JT, Leung K, Bushman M, Kishore N, Niehus R, de Salazar PM, Cowling BJ, Lipsitch M, Leung GM. Estimating clinical severity of COVID-19 from the transmission dynamics in Wuhan, China. Nat Med. 2020;26(4):506-10.

39. Khalatbari-Soltani S, Cumming RC, Delpierre C, Kelly-Irving M. Importance of collecting data on socioeconomic determinants from the early stage of the COVID-19 outbreak onwards. J Epidemiol Community Health. 2020;74(8): $620-3$.

\section{Publisher's Note}

Springer Nature remains neutral with regard to jurisdictional claims in published maps and institutional affiliations.
Ready to submit your research? Choose BMC and benefit from:

- fast, convenient online submission

- thorough peer review by experienced researchers in your field

- rapid publication on acceptance

- support for research data, including large and complex data types

- gold Open Access which fosters wider collaboration and increased citations

- maximum visibility for your research: over $100 \mathrm{M}$ website views per year

At $\mathrm{BMC}$, research is always in progress.

Learn more biomedcentral.com/submissions 